\title{
Optimal Bounds for the Neuman-Sándor Mean in terms of the Convex Combination of the First and Second Seiffert Means
}

\author{
Hao-Chuan Cui, Nan Wang, and Bo-Yong Long \\ School of Mathematical Science, Anhui University, Hefei 230601, China \\ Correspondence should be addressed to Bo-Yong Long; longboyong@ahu.edu.cn
}

Received 15 June 2015; Accepted 21 July 2015

Academic Editor: Dapeng P. Du

Copyright (C) 2015 Hao-Chuan Cui et al. This is an open access article distributed under the Creative Commons Attribution License, which permits unrestricted use, distribution, and reproduction in any medium, provided the original work is properly cited.

We find the least value $\alpha$ and the greatest value $\beta$ such that the double inequality $\alpha P(a, b)+(1-\alpha) T(a, b)<M(a, b)<\beta P(a, b)+$ $(1-\beta) T(a, b)$ holds for all $a, b>0$ with $a \neq b$, where $M(a, b), P(a, b)$, and $T(a, b)$ are the Neuman-Sándor mean and the first and second Seiffert means of two positive numbers $a$ and $b$, respectively.

\section{Introduction}

For $a, b>0$ with $a \neq b$, the Neuman-Sándor mean $M(a, b)$ [1], the first Seiffert mean $P(a, b)[2]$, and the second Seiffert mean $T(a, b)[3]$ are defined by

$$
\begin{aligned}
& M(a, b)=\frac{a-b}{2 \sinh ^{-1}((a-b) /(a+b))}, \\
& P(a, b)=\frac{a-b}{4 \tan ^{-1}(\sqrt{a / b})-\pi}, \\
& T(a, b)=\frac{a-b}{2 \tan ^{-1}((a-b) /(a+b))},
\end{aligned}
$$

respectively. It can be observed that the first Seiffert mean $P(a, b)$ can be rewritten as (see [1])

$$
P(a, b)=\frac{a-b}{2 \sin ^{-1}((a-b) /(a+b))}
$$

where $\sinh ^{-1}(x)=\log \left(x+\sqrt{x^{2}+1}\right), \tan ^{-1}(x)=\arctan (x)$, and $\sin ^{-1}(x)=\arcsin (x)$ are the inverse hyperbolic sine, inverse tangent, and inverse sine functions, respectively.

Recently, the means $M, P$, and $T$ and other means have been the subject of intensive research. Many remarkable inequalities for means can be found in the literature [4-14].
Let $H(a, b)=2 a b /(a+b), G(a, b)=\sqrt{a b}, L(a, b)=(b-$ a) $/(\log b-\log a), I(a, b)=1 / e\left(b^{b} / a^{a}\right)^{1 /(b-a)}, A(a, b)=(a+$ b) $/ 2, S(a, b)=\sqrt{\left(a^{2}+b^{2}\right) / 2}$, and

$$
M_{p}(a, b)= \begin{cases}\left(\frac{a^{p}+b^{p}}{2}\right)^{1 / p}, & p \neq 0, \\ \sqrt{a b}, & p=0\end{cases}
$$

denote the harmonic, geometric, logarithmic, identric, arithmetic, root-square, and $p$ th power means of two positive numbers $a$ and $b$ with $a \neq b$, respectively. Then it is well known that the inequalities

$$
\begin{aligned}
H(a, b) & <G(a, b)<L(a, b)<P(a, b)<I(a, b) \\
& <A(a, b)<M(a, b)<T(a, b)<S(a, b)
\end{aligned}
$$

hold for $a, b>0$ with $a \neq b$.

Neuman and Sándor [1] established

$$
\frac{\pi}{2} P(a, b)>\sinh ^{-1}(1) M(a, b)>\frac{\pi}{4} T(a, b)
$$

for all $a, b>0$ with $a \neq b$ and the following Ky Fan inequalities:

$$
\begin{aligned}
\frac{G(a, b)}{G\left(a^{\prime}, b^{\prime}\right)} & <\frac{L(a, b)}{L\left(a^{\prime}, b^{\prime}\right)}<\frac{P(a, b)}{P\left(a^{\prime}, b^{\prime}\right)}<\frac{A(a, b)}{A\left(a^{\prime}, b^{\prime}\right)} \\
& <\frac{M(a, b)}{M\left(a^{\prime}, b^{\prime}\right)}<\frac{T(a, b)}{T\left(a^{\prime}, b^{\prime}\right)}
\end{aligned}
$$

for all $0<a, b \leq 1 / 2$ with $a \neq b, a^{\prime}=1-a$ and $b^{\prime}=1-b$. 
Gao [15] proved the optimal double inequalities

$$
\begin{aligned}
\frac{e}{\pi} I(a, b) & <P(a, b)<I(a, b), \\
I(a, b) & <T(a, b)<\frac{2 e}{\pi} I(a, b)
\end{aligned}
$$

hold for all $a, b>0$ with $a \neq b$.

The following bounds for the Seiffert means $P(a, b)$ and $T(a, b)$ in terms of power mean were presented by Jagers in [16]:

$$
M_{1 / 2}<P(a, b)<M_{2 / 3}(a, b)
$$

for all $a, b>0$ with $a \neq b$. Hästö [17] improved the results of [16] and found the sharp lower power mean bound for the Seiffert mean $P(a, b)$ as follows:

$$
P(a, b)>M_{\log 2 / \log \pi}(a, b)
$$

for all $a, b>0$ with $a \neq b$.

In [18], the authors proved the sharp double inequality

$$
M_{\log 2 /(\log \pi-\log 2)}<T(a, b)<M_{5 / 3}(a, b)
$$

holds for all $a, b>0$ with $a \neq b$.

Let $\bar{L}_{p}(a, b)=\left(a^{p+1}+b^{p+1}\right) /\left(a^{p}+b^{p}\right)$ denote the Lehmer mean of two positive numbers $a$ and $b$ with $a \neq b$. In [19] the authors presented the following best possible Lehmer mean bounds for the Seiffert means $P(a, b)$ and $T(a, b)$ :

$$
\begin{aligned}
\bar{L}_{-1 / 6}(a, b) & <P(a, b)<\bar{L}_{0}(a, b), \\
\bar{L}_{0}(a, b) & <T(a, b)<\bar{L}_{1 / 3}(a, b)
\end{aligned}
$$

for all $a, b>0$ with $a \neq b$.

Let $u, v$, and $w$ be bivariate means such that $u(a, b)<$ $w(a, b)<v(a, b)$ for all $a, b>0$ with $a \neq b$. The problems of finding the best possible parameters $\alpha$ and $\beta$ such that the inequalities $\alpha u(a, b)+(1-\alpha) v(a, b)<w(a, b)<\beta u(a, b)+(1-$ $\beta) v(a, b)$ and $u(a, b)^{\alpha} v^{1-\alpha}(a, b)<w(a, b)<u(a, b)^{\beta} v^{1-\beta}(a, b)$ hold for all $a, b>0$ with $a \neq b$ have attracted the interest of many mathematicians.

In $[20,21]$ the authors proved that the double inequalities

$$
\begin{aligned}
& S(a, b)^{\alpha_{3}} A^{1-\alpha_{3}}(a, b)<M(a, b) \\
& \quad<S(a, b)^{\beta_{3}} A^{1-\beta_{3}}(a, b), \\
& \alpha_{4} S(a, b)+\left(1-\alpha_{4}\right) G(a, b)<M(a, b) \\
& \quad<\beta_{4} S(a, b)+\left(1-\beta_{4}\right) G(a, b)
\end{aligned}
$$

hold for all $a, b>0$ with $a \neq b$ if and only if $\alpha_{3} \leq 1 / 3, \beta_{3} \geq$ $2(\log (2+\sqrt{2})-\log 3) / \log 2, \alpha_{4} \leq 2 / 3$, and $\beta_{4} \geq 1 /[\sqrt{2} \log (1+$ $\sqrt{2})]$.

In [22], the authors proved that the double inequality

$$
\begin{gathered}
\alpha_{5} A(a, b)+\left(1-\alpha_{5}\right) G(a, b)<P(a, b) \\
<\beta_{5} A(a, b)+\left(1-\beta_{5}\right) G(a, b)
\end{gathered}
$$

holds for all $a, b>0$ with $a \neq b$ if and only if $\alpha_{5} \leq \pi / 2$, $\beta_{5} \geq 2 / 3$.

The main purpose of this paper is to find the least value $\alpha$ and the greatest value $\beta$ such that the double inequality

$$
\begin{gathered}
\alpha P(a, b)+(1-\alpha) T(a, b)<M(a, b) \\
<\beta P(a, b)+(1-\beta) T(a, b)
\end{gathered}
$$

holds for all $a, b>0$ with $a \neq b$.

\section{Lemmas}

To establish our main result, we need several lemmas which we present in this section.

Lemmas 1 and 2 were proved in [23].

Lemma 1. Let $H(x)=x / \sqrt{x^{2}+1} \sinh ^{-1}(x)^{2}-1 / \sinh ^{-1}(x)$. Then $H(x)$ is strictly decreasing on $(0,1)$. Moreover, the inequality

$$
H(x)>-\frac{x}{3}+\frac{x^{3}}{9}
$$

holds for any $x \in(0,0.7)$ and the inequality

$$
H(x)<-\frac{x}{3}+\frac{17 x^{3}}{90}
$$

holds for any $x \in(0,1)$.

Lemma 2. Let $S(x)=1 / \sin ^{-1}(x)-x / \sqrt{1-x^{2}}\left(\sin ^{-1}(x)\right)^{2}$. Then it holds that

$$
S(x)>-\frac{x}{3}-\frac{x^{3}}{3}
$$

for any $x \in(0,0.7)$ and

$$
S(x)<-\frac{x}{3}-\frac{17 x^{3}}{90}-\frac{x^{5}}{7}
$$

holds for any $x \in(0,1)$.

For $x \in(0,1)$, the power series expansion of the function $\tan ^{-1}(x)$ is presented as follows:

$$
\tan ^{-1}(x)=x-\frac{x^{3}}{3}+\frac{x^{5}}{5}+\cdots=\sum_{n=0}^{\infty} \frac{(-1)^{n}}{2 n+1} x^{2 n+1}
$$

Therefore, we have the following.

Lemma 3. If $x \in(0,1)$, then it holds that

$$
\begin{aligned}
x-\frac{x^{3}}{3} & <\tan ^{-1}(x)<x-\frac{x^{3}}{3}+\frac{x^{5}}{5}<x, \\
\tan ^{-1}(x) & <x-\frac{x^{3}}{5}
\end{aligned}
$$


Proof. The inequality of (22) follows from equality (21) directly. The inequality of (23) follows from

$$
\begin{aligned}
& {\left.\left[\tan ^{-1}(x)-x+\frac{x^{3}}{5}\right]\right|_{x=0}=0} \\
& {\left[\tan ^{-1}(x)-x+\frac{x^{5}}{5}\right]^{\prime}=-x^{2}\left(\frac{1}{1+x^{2}}+\frac{3 x^{2}}{5}\right)<0}
\end{aligned}
$$

for $x \in(0,1)$.

Lemma 4. Let $T(x)=1 / \tan ^{-1}(x)-x /\left(x^{2}+1\right) \tan ^{-1}(x)^{2}$. Then for $x \in(0,1)$ it holds that

$$
\frac{2 x}{3}-\frac{2 x^{3}}{5}+\frac{x^{5}}{7}<T(x)<\frac{2 x}{3}-\frac{2 x^{3}}{9}+\frac{2 x^{5}}{7} .
$$

Proof. To prove inequality (25), it suffices to show

$$
\begin{aligned}
& t_{1}(x)>0, \\
& t_{2}(x)<0
\end{aligned}
$$

for $x \in(0,1)$, where

$$
\begin{aligned}
& t_{1}(x) \\
& =\left(1+x^{2}\right)\left(\tan ^{-1}(x)\right)^{2}\left[T(x)-\frac{2 x}{3}+\frac{2 x^{3}}{5}-\frac{x^{5}}{7}\right], \\
& t_{2}(x) \\
& =\left(1+x^{2}\right)\left(\tan ^{-1}(x)\right)^{2}\left[T(x)-\frac{2 x}{3}+\frac{2 x^{3}}{9}-\frac{2 x^{5}}{7}\right] .
\end{aligned}
$$

Firstly, we prove inequality (26). From the expression of $t_{1}(x)$, we have

$$
\begin{aligned}
& t_{1}(0)=0, \\
& t_{1}^{\prime}(x)=\tan ^{-1}(x) t_{1}^{*}(x),
\end{aligned}
$$

where

$$
\begin{aligned}
t_{1}^{*}(x)= & \frac{2 x}{3}+\frac{4 x^{3}}{5}-\frac{2 x^{5}}{7} \\
& -\tan ^{-1}(x)\left[\frac{2}{3}+\frac{4 x^{2}}{5}-\frac{9 x^{4}}{7}+x^{6}\right] .
\end{aligned}
$$

Because of

$$
\begin{aligned}
\frac{2}{3}+\frac{4 x^{2}}{5}-\frac{9 x^{4}}{7}+x^{6} & >\frac{2 x^{4}}{3}+\frac{4 x^{4}}{5}-\frac{9 x^{4}}{7}+x^{6} \\
& =\frac{19 x^{4}}{105}+x^{6}>0
\end{aligned}
$$

for $x(0,1)$, it follows from (22) and (31) that

$$
\begin{aligned}
t_{1}^{*}(x)> & \frac{2 x}{3}+\frac{4 x^{3}}{5}-\frac{2 x^{5}}{7} \\
& -x\left(\frac{2}{3}+\frac{4 x^{2}}{5}-\frac{9 x^{4}}{7}+x^{6}\right)=x^{5}-x^{7}>0
\end{aligned}
$$

for any $x \in(0,1)$. Therefore, inequality (26) follows from (29) and (30) together with (33).

Secondly, we prove inequality (27). From the expression of $t_{2}(x)$, we have

$$
\begin{aligned}
t_{2}(0) & =0, \\
t_{2}^{\prime} & =\tan ^{-1}(x) t_{2}^{*}(x),
\end{aligned}
$$

where

$$
\begin{aligned}
t_{2}^{*}(x)= & \frac{2 x}{3}+\frac{4 x^{3}}{9}-\frac{4 x^{5}}{7} \\
& -\tan ^{-1}(x)\left(\frac{2}{3}+\frac{4 x^{2}}{3}+\frac{20 x^{4}}{63}+2 x^{6}\right) .
\end{aligned}
$$

Because of

$$
\frac{2}{3}+\frac{4 x^{2}}{3}+\frac{20 x^{4}}{63}+2 x^{6}>0
$$

for any $x \in(0,1)$, it follows from (22) and (35) together with (36) that

$$
\begin{aligned}
t_{2}^{*}(x)< & \frac{2 x}{3}+\frac{4 x^{3}}{9}-\frac{4 x^{5}}{7} \\
& -\left(x-\frac{x^{3}}{3}\right)\left(\frac{2}{3}+\frac{4 x^{2}}{3}+\frac{20 x^{4}}{63}+2 x^{6}\right) \\
= & -\frac{2 x^{3}}{3}-\frac{4 x^{5}}{9}-\frac{358 x^{7}}{189}+\frac{2 x^{9}}{3} \\
< & -\frac{2 x^{3}}{3}-\frac{4 x^{5}}{9}-\frac{358 x^{7}}{189}+\frac{2 x^{7}}{3}<-\frac{2 x^{3}}{3}-\frac{4 x^{5}}{9} \\
< & 0
\end{aligned}
$$

for any $x \in(0,1)$. Therefore, inequality (27) follows from (34) and (37).

Lemma 5. The function $f(x)=\lambda S(x)+(1-\lambda) T(x)+H(x)$ is strictly decreasing on $(0.7,1)$, where $\lambda=(1 / 2) \pi(4 / \pi-$ $\left.1 / \sinh ^{-1}(1)\right)=0.2177 \ldots$ and $H(x), S(x)$, and $T(x)$ are defined as in Lemmas 1, 2, and 4, respectively.

Proof. Direct computations lead to

$$
\begin{aligned}
T^{\prime}(x) & =2 \frac{x-\tan ^{-1}(x)}{\left(1+x^{2}\right)^{2}\left(\tan ^{-1}(x)\right)^{2}}, \\
T^{\prime \prime}(x) & =2 \frac{\psi(x)}{\left(1+x^{2}\right)^{3}\left(\tan ^{-1}\right)^{4}},
\end{aligned}
$$

where

$$
\psi(x)=-3 x+3\left(1-x^{2}\right) \tan ^{-1}+4 x\left(\tan ^{-1}(x)\right)^{2} .
$$


Then inequality (22) leads to

$$
\begin{aligned}
\psi(x)< & -3 x+3\left(1-x^{2}\right)\left(x-\frac{x^{3}}{3}+\frac{x^{5}}{5}\right) \\
& +4 x\left(x-\frac{x^{3}}{3}+\frac{x^{5}}{5}\right)^{2} \\
= & \frac{4}{25} x^{3}\left[x^{4}-\frac{25 x^{2}}{4}+\frac{5}{2}\right]
\end{aligned}
$$

for any $x \in(0.7,1)$. Obviously, it holds that

$$
x^{4}-\frac{25 x^{2}}{4}+\frac{5}{2}<0.7^{4}-\frac{25}{4} 0.7^{2}+\frac{5}{2}=-0.3224<0
$$

for any $x \in(0.7,1)$. Therefore, for any $x \in(0.7,1), T^{\prime \prime}(x)<0$ follows from (39) and (41) together with (42). In other words, $T^{\prime}(x)$ is strictly decreasing on $(0.7,1)$.

Let $\phi(x)=\lambda S(x)+(1-\lambda) T(x)$. It was proved that $S^{\prime}(x)$ is strictly decreasing on $(0.7,1)$ in [23] Lemma 2.4 . Thus, from the monotonicity of $S^{\prime}(x)$ and $T^{\prime}(x)$, we have

$$
\begin{aligned}
\phi^{\prime}(\lambda, x) & <\lambda S^{\prime}(0.7)+(1-\lambda) T^{\prime}(0.7)=-0.0507 \cdots \\
& <0
\end{aligned}
$$

for any $x \in(0.7,1)$. That is to say, $\phi(x)$ is strictly decreasing on $(0.7,1)$. Considering the monotonicity of $H(x)$ in Lemma 1 , the proof is completed.

Lemma 6. Let $\xi(x)=x\left[((1-\lambda) / 7) x^{4}-(13 / 45-\lambda / 15) x^{2}+\right.$ $(1 / 3-\lambda)]$, where $\lambda=(1 / 2) \pi\left(4 / \pi-1 / \sinh ^{-1}(1)\right)=0.2177 \ldots$. Then $\xi(x)>0$ for $x \in(0,0.7)$.

Proof. Let $\zeta(x)=((1-\lambda) / 7) x^{4}-(13 / 45-\lambda / 15) x^{2}+(1 / 3-\lambda)$. Then it is easy to verify that $\zeta(x)$ is decreasing on $(0, \mu)$, where

$$
\mu=\frac{1}{3} \sqrt{\frac{7\left(3 \pi+14 \sinh ^{-1}(1)\right)}{10\left(\pi-2 \sinh ^{-1}(1)\right)}}=1.1079 \ldots
$$

Considering $\zeta(0.7)=0.0079 \ldots>0$, we have $\zeta(x)>0$ for $x \in(0,0,7)$. Therefore, $\xi(x)>0$ for $x \in(0,0,7)$.

\section{Main Results}

Theorem 7. The double inequality

$$
\begin{gathered}
\alpha P(a, b)+(1-\alpha) T(a, b)<M(a, b) \\
<\beta P(a, b)+(1-\beta) T(a, b)
\end{gathered}
$$

holds for any $a, b>0$ with $a \neq b$ if and only if $\alpha \geq 1 / 3$ and $\beta \leq(1 / 2) \pi\left(4 / \pi-1 / \sinh ^{-1}(1)\right)=0.2177 \ldots$.

Proof. Because $P(a, b), M(a, b)$, and $T(a, b)$ are symmetric and homogeneous of degree 1 , without loss of generality, we assume that $a>b$. Let $p \in(0,1), x=(a-b) /(a+b) \in(0,1)$, and $\lambda=(1 / 2) \pi\left(4 / \pi-1 / \sinh ^{-1}(1)\right)$. Then by (1), (3), and (4), direct computations lead to

$$
\begin{aligned}
& \frac{P(a, b)}{A(a, b)}=\frac{x}{\sin ^{-1}(x)}, \\
& \frac{M(a, b)}{A(a, b)}=\frac{x}{\sinh ^{-1}(x)}, \\
& \frac{T(a, b)}{A(a, b)}=\frac{x}{\tan ^{-1}(x)} .
\end{aligned}
$$

Let

$$
\begin{aligned}
D_{p}(x):= & \frac{p P(a, b)+(1-p) T(a, b)-M(a, b)}{A(a, b)} \\
= & p \frac{x}{\sin ^{-1}(x)}+(1-p) \frac{x}{\tan ^{-1}(x)} \\
& -\frac{x}{\sinh ^{-1}(x)} .
\end{aligned}
$$

Then it follows that

$$
\begin{aligned}
& D_{p}\left(0^{+}\right)=0, \\
& D_{p}\left(1^{-}\right)=\frac{4}{\pi}-\frac{2 p}{\pi}-\frac{1}{\sinh ^{-1}(1)}, \\
& D_{\lambda}\left(1^{-}\right)=0 .
\end{aligned}
$$

Differentiating $D_{p}(x)$, we have

$$
\begin{aligned}
& D_{p}^{\prime}(x) \\
& =p\left[\frac{1}{\sin ^{-1}(x)}-\frac{x}{\sqrt{1-x^{2}}\left(\sin ^{-1}(x)\right)^{2}}\right] \\
& \quad+(1-p)\left[\frac{1}{\tan ^{-1}(x)}-\frac{x}{\left(x^{2}+1\right) \tan ^{-1}(x)^{2}}\right] \\
& \quad-\left[\frac{x}{\sqrt{x^{2}+1} \sinh ^{-1}(x)^{2}}-\frac{1}{\sinh ^{-1}(x)}\right] \\
& =p S(x)+(1-p) T(x)+H(x),
\end{aligned}
$$

where $H(x), S(x)$, and $T(x)$ are defined as in Lemmas 1, 2, and 4 , respectively.

On one hand, from inequalities in Lemmas 1, 2, and 4, we clearly see that

$$
\begin{aligned}
D_{1 / 3}^{\prime}(x)= & \frac{1}{3} S(x)+\frac{2}{3} T(x)+H(x) \\
< & \frac{1}{3}\left(-\frac{x}{3}-\frac{17 x^{3}}{90}-\frac{x^{5}}{7}\right) \\
& +\frac{2}{3}\left(\frac{2 x}{3}-\frac{2 x^{3}}{9}+\frac{2 x^{5}}{7}\right)-\frac{x}{3}+\frac{17 x^{3}}{90} \\
= & -\frac{2}{9} x-\frac{4}{24} x^{3}+\frac{1}{27} x^{5}<-\frac{2}{9} x<0
\end{aligned}
$$


for $x \in(0,1)$. It leads to

$$
D_{1 / 3}(x)<D_{1 / 3}(0)=0
$$

for any $x \in(0,1)$. According to (47) and (51), we conclude that

$$
M(a, b)>\frac{1}{3} P(a, b)+\frac{2}{3} T(a, b)
$$

for all $a, b>0$ with $a \neq b$.

On the other hand, from inequalities in Lemmas 1, 2, and 4, we have

$$
\begin{aligned}
D_{\lambda}^{\prime}(x) & \\
> & -\lambda\left(\frac{x}{3}+\frac{x^{3}}{3}\right)+(1-\lambda)\left(\frac{2 x}{3}-\frac{2 x^{3}}{5}+\frac{x^{5}}{7}\right) \\
& -\frac{x}{3}+\frac{x^{3}}{9} \\
= & x\left[\left(\frac{1-\lambda}{7}\right) x^{4}-\left(\frac{13}{45}-\frac{\lambda}{15}\right) x^{2}+\left(\frac{1}{3}-\lambda\right)\right]
\end{aligned}
$$

for $(0,0.7)$. According to Lemma 6, we have

$$
D_{\lambda}^{\prime}(x)>\xi(x)>0
$$

for $(0,0.7)$. Lemma 5 shows that $D_{\lambda}^{\prime}(x)$ is strictly decreasing on $(0.7,1)$. This fact and (54) together with $D_{\lambda}^{\prime}\left(1^{-}\right)=$ $-\infty$ imply that there exists $x_{0} \in(0.7,1)$ such that $D_{\lambda}(x)$ is strictly increasing on $\left(0, x_{0}\right]$ and strictly decreasing on $\left[x_{0}, 1\right)$. Equations (47) and (48) together with the piecewise monotonicity of $D_{\lambda}(x)$ lead to the conclusion that

$$
M(a, b)<\lambda P(a, b)+(1-\lambda) T(a, b)
$$

for all $a, b>0$ with $a \neq b$.

At last, by easy computations, (1), (3), and (4) lead to

$$
\begin{gathered}
\frac{T(a, b)-M(a, b)}{T(a, b)-P(a, b)}=\frac{x / \tan ^{-1}(x)-x / \sinh ^{-1}(x)}{x / \tan ^{-1}(x)-x / \sin ^{-1}(x)}, \\
\lim _{x \rightarrow 0^{+}} \frac{x / \tan ^{-1}(x)-x / \sinh ^{-1}(x)}{x / \tan ^{-1}(x)-x / \sin ^{-1}(x)}=\frac{1}{3}, \\
\lim _{x \rightarrow 1^{-}} \frac{x / \tan ^{-1}(x)-x / \sinh ^{-1}(x)}{x / \tan ^{-1}(x)-x / \sin ^{-1}(x)}=\lambda .
\end{gathered}
$$

Thus, we have the following claims.

Claim 1. If $\alpha<1 / 3$, then (56) and (57) imply that there exists $\sigma_{1} \in(0,1)$ such that $M(a, b)<\alpha P(a, b)+(1-\alpha) T(a, b)$ for all $a, b$ with $(a-b) /(a+b) \in\left(0, \sigma_{1}\right)$.

Claim 2. If $\beta>\lambda$, then (56) and (58) imply that there exists $\sigma_{2} \in(0,1)$ such that $M(a, b)>\beta P(a, b)+(1-\beta) T(a, b)$ for all $a, b$ with $(a-b) /(a+b) \in\left(1-\sigma_{2}, 1\right)$.

Inequalities (52) and (55) in conjunction with the above two claims mean the proof is completed.

\section{Conflict of Interests}

The authors declare that they have no competing interests.

\section{Acknowledgments}

This research was supported by the Program of Scientific Research Training for Undergraduate of Anhui University (KYXL2014002) and Doctoral Scientific Research Foundation of Anhui University, China (01001901).

\section{References}

[1] E. Neuman and J. Sándor, "On the Schwab-Borchardt mean," Mathematica Pannonica, vol. 14, no. 2, pp. 253-266, 2003.

[2] H.-J. Seiffert, "Problem 887," Nieuw Archief voor Wiskunde, vol. 11, no. 4, p. 176, 1993.

[3] H.-J. Seiffert, "Aufgabe $\beta$ 16," Die Wurzel, vol. 29, pp. 221-222, 1995.

[4] J. M. Borwein and P. B. Borwein, "Inequalities for compound mean iterations with logarithmic asymptotes," Journal of Mathematical Analysis and Applications, vol. 177, no. 2, pp. 572-582, 1993.

[5] P. S. Bullen, D. S. Mitrinović, and P. M. Vasić, Means and Their inequalities, D. Reidel Publishing, Dordrecht, The Netherlands, 1988.

[6] H. Liu and X.-J. Meng, "The optimal convex combination bounds for Seiffert's mean," Journal of Inequalities and Applications, vol. 2011, Article ID 686834, 9 pages, 2011.

[7] E. Neuman, "On generalized Seiffert means," Aequationes Mathematicae, vol. 87, no. 3, pp. 325-335, 2014.

[8] E. Neuman and J. Sándor, "On certain means of two arguments and their extensions," International Journal of Mathematics and Mathematical Sciences, no. 16, pp. 981-993, 2003.

[9] F. Qi and W.-H. Li, "A unified proof of several inequalities and some new inequalities involving Neuman-Sndor mean," Miskolc Mathematical Notes, vol. 15, no. 2, pp. 665-675, 2014.

[10] J. Sándor, “On certain inequalities for means III," Archiv der Mathematik, vol. 76, no. 1, pp. 30-40, 2001.

[11] H.-J. Seiffert, "Ungleichunen für einen bestimmten Mittelwert," Nieuw Archief voor Wiskunde, vol. 13, no. 2, pp. 195-198, 1995.

[12] M. K. Vamanamurthy and M. Vuorinen, "Inequalities for means," Journal of Mathematical Analysis and Applications, vol. 183, no. 1, pp. 155-166, 1994.

[13] A. Witkowski, "Optimal weighted harmonic interpolations between Seiffert means," Colloquium Mathematicum, vol. 130, no. 2, pp. 265-279, 2013.

[14] Z.-H. Yang, "Estimates for Neuman-Sándor mean by power means and their relative errors," Journal of Máthematical Inequalities, vol. 7, no. 4, pp. 711-726, 2013.

[15] S.-Q. Gao, "Inequalities for the Seiffert's means in term of the identic mean," Journal of Advanced Mathematics and Applications, vol. 10, no. 1-2, pp. 23-31, 2011.

[16] A. A. Jagers, "Solution of problem 887," Nieuw Archief voor Wiskunde, vol. 12, no. 4, pp. 230-231, 1994.

[17] P. A. Hästö, "Optimal inequalities between Seiffert's mean and power means," Mathematical Inequalities \& Applications, vol. 7, no. 1, pp. 47-53, 2004.

[18] Y.-M. Li, M.-K. Wang, and Y.-M. Chu, "Sharp power mean bounds for Seiffert mean," Applied Mathematics-Serie B, vol. 29, no. 1, pp. 101-107, 2014. 
[19] M.-K. Wang, Y.-F. Qiu, and Y.-M. Chu, "Sharp bounds for Seiffert means in terms of Lehmer means," Journal of Mathematical Inequalities, vol. 4, no. 4, pp. 581-586, 2010.

[20] E. Neuman, "A note on a certain bivariate mean," Journal of Mathematical Inequalities, vol. 6, no. 4, pp. 637-643, 2012.

[21] T.-H. Zhao, Y.-M. Chu, and B.-Y. Liu, "Optimal bounds for Neuman-Sándor mean in terms of the convex combinations of harmonic, geometric, quadratic, and contraharmonic means," Abstract and Applied Analysis, vol. 2012, Article ID 302635, 9 pages, 2012.

[22] W.-D. Jiang and F. Qi, "Some sharp inequalities involving Seiffert and other means and their concise proofs," Mathematical Inequalities \& Applications, vol. 15, no. 4, pp. 1007-1017, 2012.

[23] Y. Chu, T. Zhao, and Y. Song, "Sharp bounds for NeumanSándor mean in terms of the convex combination of quadratic and first SEIffert means," Acta Mathematica Scientia-Series B, vol. 34, no. 3, pp. 797-806, 2014. 


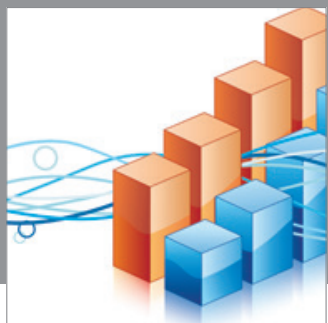

Advances in

Operations Research

mansans

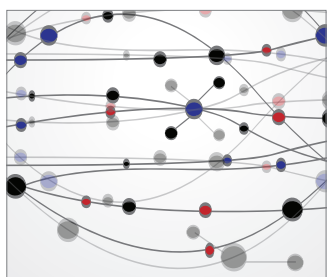

The Scientific World Journal
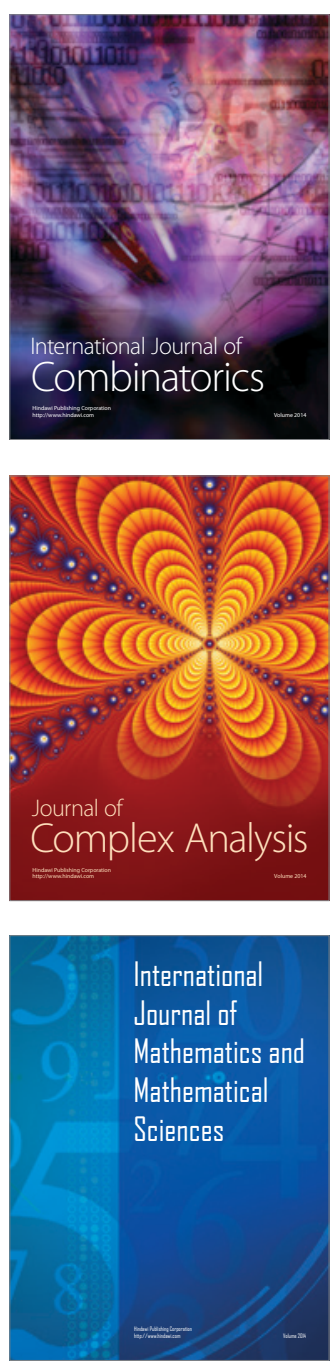
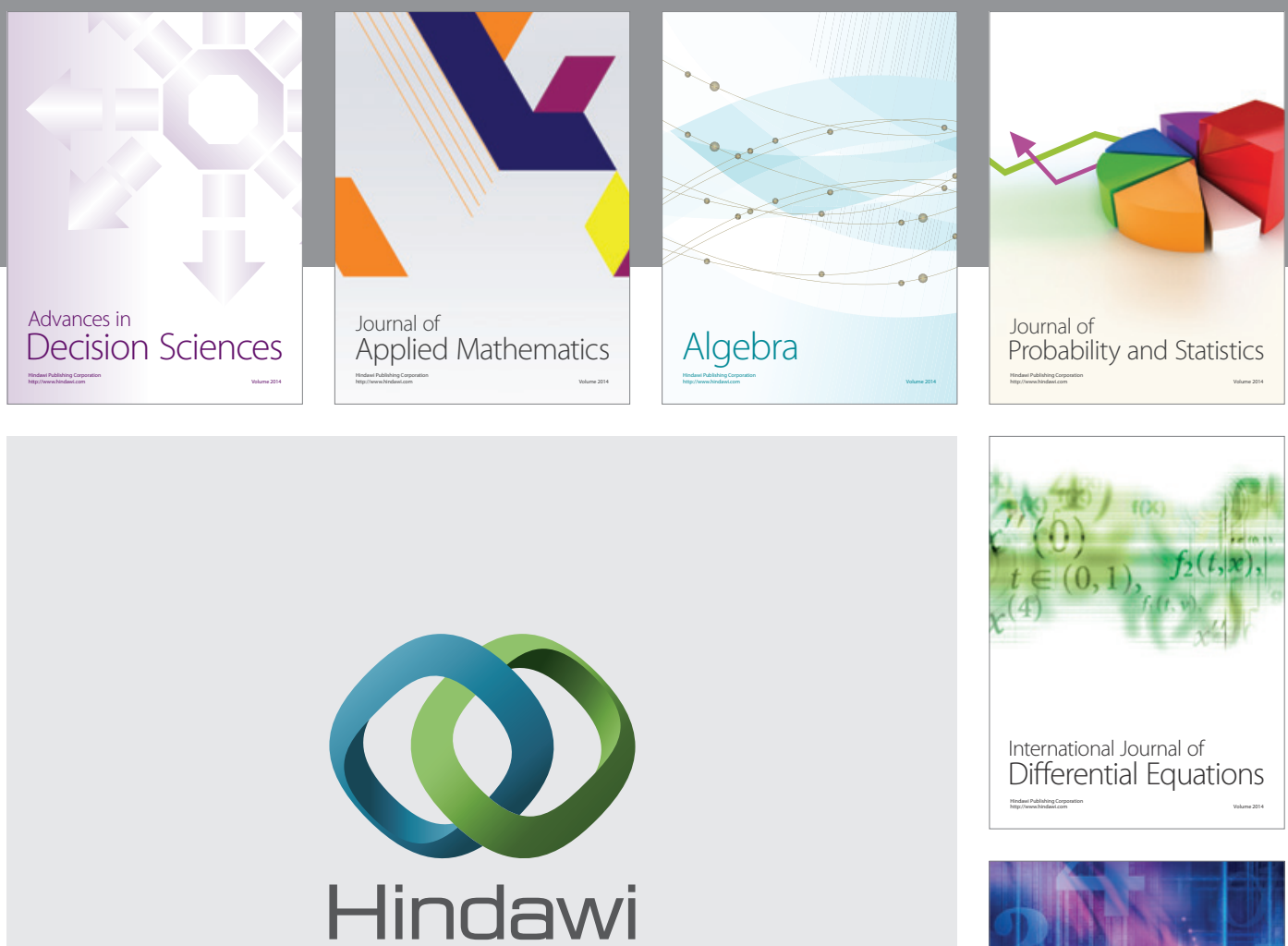

Submit your manuscripts at http://www.hindawi.com
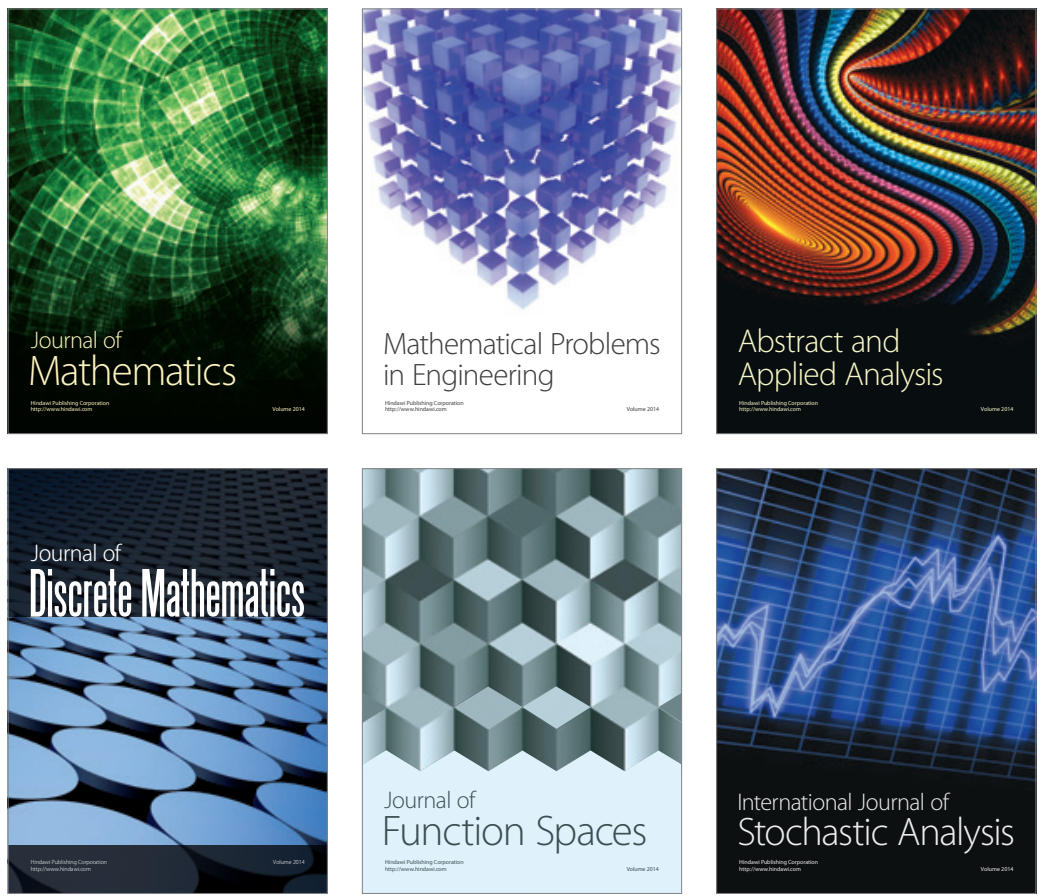

Journal of

Function Spaces

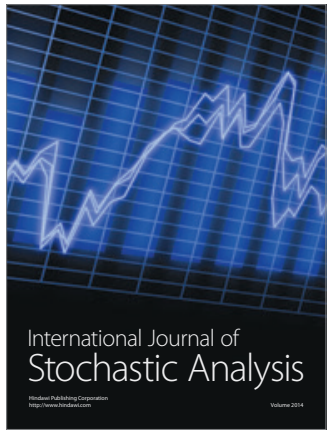

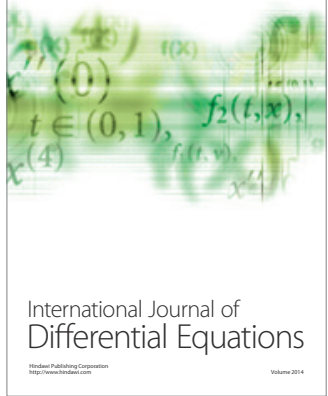
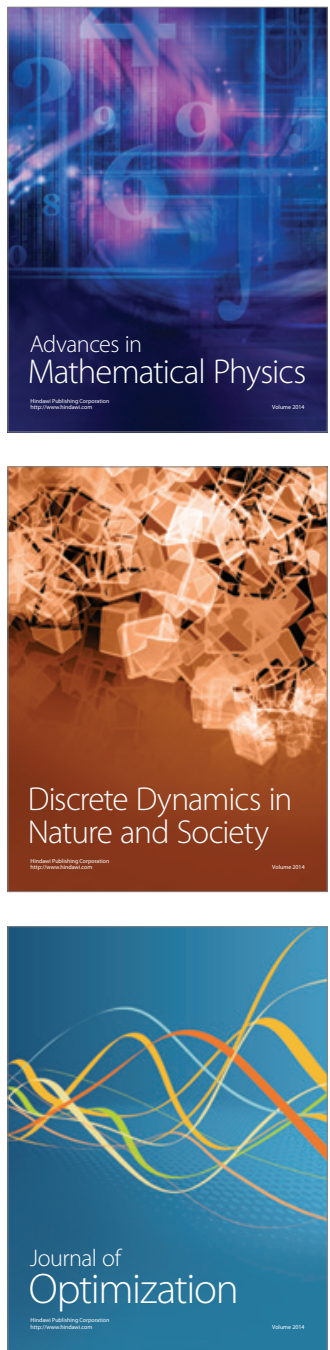